\title{
Hydatid Disease of liver communicating with common bile duct managed by ERCP - A case report
}

\author{
Anurag Jha*, Rahul Pathak, Sashi Sharma, Prem Khadga \\ Department of Gastoenterology, Institute of Medicine, Tribhuvan University, Kathmandu, Nepal
}

DOI Name

http://dx.doi.org/10.3126/jaim.v5i2.17352

\section{Keywords}

Cholangitis; Hydatid cysts; Biliary Obstruction:

ERCP

\section{Citation}

Anurag Jha, Rahul Pathak, Sashi Sharma, Prem Khadga. Hydatid disease of liver communicating with common bile duct managed by ERCP - A case report. Journal of Advances in Internal Medicine 2016;05(02):38-40.

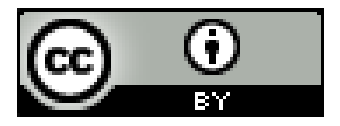

This work is licensed under a Creative Commons Attribution 3.0 Unported License.

\begin{abstract}
Cystic hepatic disease is common in Asia, South America and Africa. Rupture of the hydatid cyst into the biliary tree can lead to serious cholangitis. In this report, a 53-year-old lady presented with the signs and symptoms of obstructive jaundice and cholangitis. Ultrasonography reported dilated common bile duct(CBD) with sludge and hydatid cysts in the liver. CECT revealed large cysts with enhancing thin wall in left and right lobe of liver with communicating into $C B D$ and right IHBD respectively and features of obstructive biliopathy. Due to signs and symptoms of obstructive jaundice in addition to lab data and imaging modalities, ruptured hydatid cyst into the biliary tree was considered, and ERCP intervention was performed, after endoscopic sphincterotomy, membranes of the cysts were extracted. Post intervention, signs and symptoms and cholestasis enzymes subsided.
\end{abstract}

\section{INTRODUCTION}

Hepatic echinococcosis, also called hepatic hydatid cyst (HHC) is common in the Middle East, India, Nepal and Africa. It is a zoonosis that although endemic mostly in sheep-farming countries, remains a public health issue worldwide, involving mainly the liver.(1) It usually occurs as a result of infection by larval stage of species E. granulosus, genus Echinococcus, via the ingestion of food and water which is fecally contaminated with eggs from the definitive hosts- dogs and wolves. Humans are an accidental intermediate host of the infection.(2) HHC is a slow developmental parasite which has various pathological lesions, responsible for different clinical pictures. The aim of the treatment is to eliminate the parasite, and to tackle the problem of the residual cavity and any other associated complications (3) The main complications of HHC are infection of the cyst, liver abscess, and rupture into either the peritoneum, biliary tree or pleural cavity, lung or pericardium. (4). Of these, intrabiliary rupture of $\mathrm{HHC}$ is the most common and grave complication, occurring in $2 \%-42 \%$ of cases (5Dolay)

\section{CASE REPORT}

A 53-year-old lady was admitted to emergency department with swelling in and pain in right upper abdominal quadrant, and jaundice for 10 days prior to admission. In addition, the patient had fever, nausea, vomiting, anorexia, and dark urine for same duration. She had been diagnosed 1 year back with HHC and treated medically. She didn't have any other GI problems, hepatitis or history of trauma, IV drug use, and transfusion. She didn't give any history of alcohol usage, and her family history was not significant. At admission, she was noted to have a temperature of $38^{\circ} \mathrm{C}$, blood pressure of $110 / 70 \mathrm{~mm} / \mathrm{Hg}$, heart rate of $100 / \mathrm{min}$ and respiratory rate of $18 / \min$.

\footnotetext{
* Corresponding author

Anurag Jha

Gastroenterology Fellow; IOM, TUTH, Nepal.

E-mail: drjhanurag@gmail.com
} 

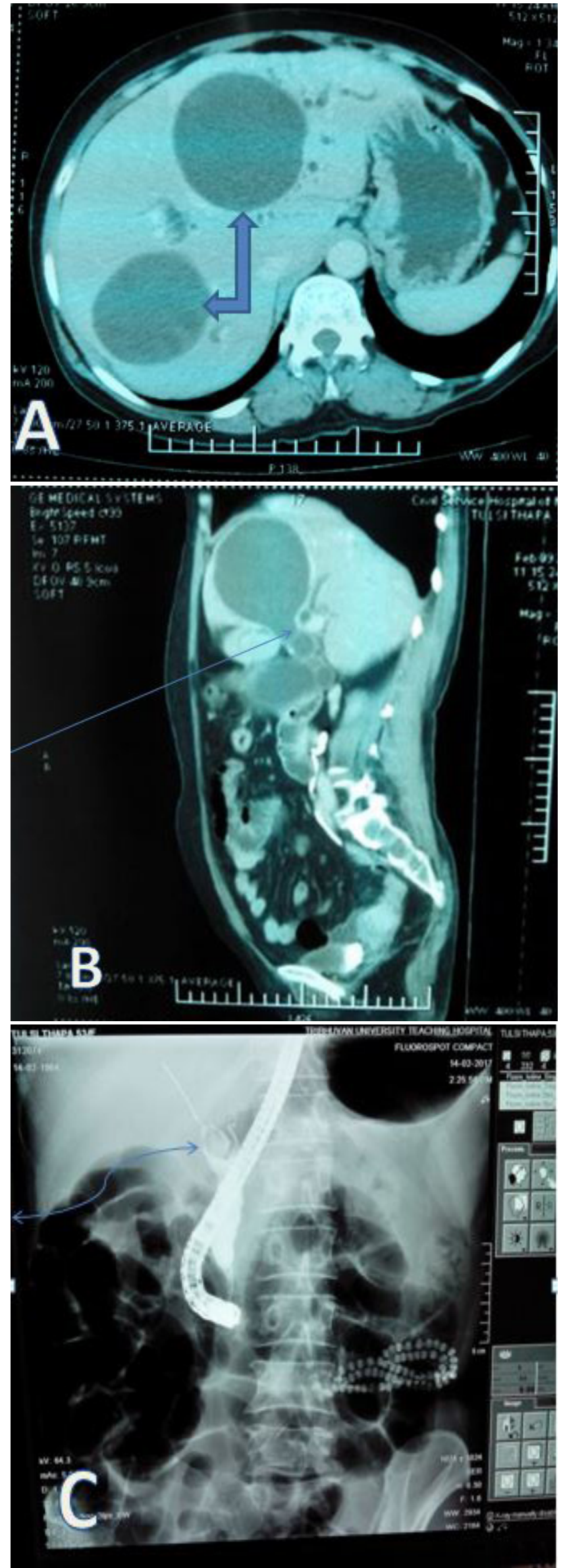

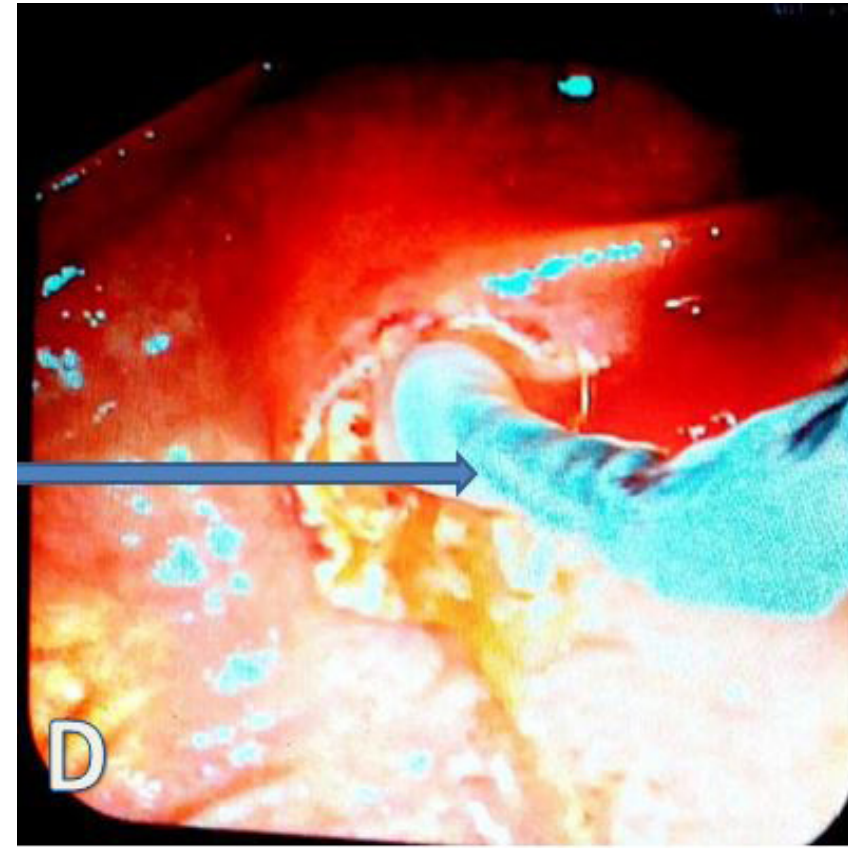

Fig A- CT scan showing two hepatic cysts (double block arrow head). Fig B- CT scan showing Hepatic cyst communicating with the $\mathrm{CBD}$ ( single arrow head). Fig C-ERCP image showing cyst membranes in $\mathrm{CBD}$ (two way arrow). Fig D- Endoscopic view of cystic membranes being extracted.

Physical examination showed icterus, tenderness in epigastrium and RUQ without rebound tenderness, murphy sign was negative. Liver was palpable $4 \mathrm{~cm}$ below right subcostal margin. The other part of the abdomen was soft, and the remainder of her examination was unremarkable. Lab data revealed $\mathrm{Hb}$ $11.1 \mathrm{gm} / \mathrm{dl}$, TLC- 16000 , direct hyperbilirubinemia T-bili:11mg/ dl, D-bili:10.2mg/dl, Alkaline phosphatase was $1996 \mathrm{U} / \mathrm{l}, \mathrm{ALT}: 70$ $\mathrm{U} / \mathrm{I}, \mathrm{AST}: 85 \mathrm{U} / \mathrm{I}$. Other findings including urea, creatinine, amylase, electrolytes, coagulation test, and albumin were normal. Ultrasound showed mild thickening of gallbladder wall, with multiple cystic lesions in liver with suspicion of hydatid cyst, and dilated CBD .CECT abdomen revealed approximately $75 \times 70 \times 60 \mathrm{~mm}$ sized well defined cyst noted in left lobe of liver and the cyst is communicating directly with the CBD inferiorly. CBD was mildly dilated- $19 \mathrm{~mm}$ in diameter Mild to moderate dilatation of bilateral intrahepatic biliary ducts. Approximately $66 \times 60 \times 60 \mathrm{~mm}$ size well defined cyst was also noted in right lobe of liver which was communicating with right intrahepatic duct inferiorly. Hydatid serology was positive.

Diagnosis of acute cholangitis was made, and it was suspected that biliary obstruction was directly caused by hydatid cyst rupture with debris and daughter cyst blocking the CBD. Patient underwent diagnostic and therapeutic ERCP with endoscopic sphincterotomy wherein the blockade caused by daughter 
cysts were removed and antihelminthic therapy started. Post ERCP intervention, the patient was free of symptoms with improvement in her LFT's.

\section{DISCUSSION:}

Cystic lesion of the liver include simple cysts, cystadenoma, cystadenocarcinoma, polycystic liver disease, liver metastasis and hydatid cysts. Hepatic hydatid disease usually is asymptomatic and after a long asymptomatic course become symptomatic in an unknown percentage of patients. Hydatid cysts of the liver enlarge progressively and produce signs and symptoms as a result of increased pressure, infection, increase in liver size due to replacement of liver or rupture into the abdominal cavity or the biliary tree.(6)

One of the most common and serious complications of hepatic hydatid disease is the rupture of the cyst into intrahepatic bile ducts. Cyst growth is expansive, involving a concentric increase in size, and can create pressures of up to $80 \mathrm{~cm} \mathrm{H}_{2} \mathrm{O}$.

(7) The clinical presentation of intrabiliary rupture can range

\section{REFERENCES:}

1. Manouras A, Genetzakis M, Antonakis PT, Lagoudianakis

E, Pattas M, Papadima A, et al. Endoscopic management of a relapsing hepatic hydatid cyst with intrabiliary rupture: a case report and review of the literature. Can J Gastroenterol. 2007 Apr;21(4):249-53.

2. Kwon BW, Park SJ, Kong JH, Song IH. Daughter cysts in a cyst of the liver: hepatic echinococcosis. Korean J Intern Med. 2016 Jan;31(1):197-8.

3. Ammar M, Hamdi A. [Hydatid cyst of the liver: report of an unusual case]. Pan Afr Med J. 2015;20:295.

4. Nemati Honar B, Hayatollah G, Nikshoar M, Forootan M, Feizi AM. Liver Hydatid Cyst and Acute Cholangitis: a Case Report. Acta Med Iran. 2016 Apr;54(4):286-8.

5. Dolay K, Akbulut S. Role of endoscopic retrograde from asymptomatic to jaundice, cholecystitis, cholangitis, liver abscess, pancreatitis and septicemia, anaphylaxis and even death. Currently, most of the complications in both the preand postoperative periods are diagnosed and treated by noninvasive or minimally invasive methods. In clinical practice, endoscopic retrograde cholangiopancreatography (ERCP) is indicated for patients with preoperative frank intrabiliary rupture in which hydatid elements are clearly seen in the bile ducts by radiological evidence, or for biliary adverse events after surgery, including persistent biliary fistulae and jaundice (5).

ERCP provides more comprehensive information. It can show impacted daughter cysts in the CBD, ampulla of Vater, displacement and distortion of the intra hepatic duct. ERCP is an alternative and effective method of treatment for hepatic hydatid cyst with biliary fistula.(8) Although it is not frequent to have a rupture of the liver hydatid cyst into the bile duct, but in patients with the evidence of liver hydatid cyst and sign and symptom of acute cholangitis, it must be considered.

cholangiopancreatography in the management of hepatic hydatid disease. World J Gastroenterol. 2014 Nov 07;20(41):15253-61

6. Goumas K, Poulou A, Dandakis D, Tyrmpas I, Georgouli $A$, Sgourakis $G$, et al. Role of endoscopic intervention in biliary complications of hepatic hydatid cyst disease. Scand J Gastroenterol. 2007 Sep;42(9):1113-9.

7.Bektas M, Dokmeci A, Cinar K, Halici I, Oztas E, Karayalcin $S$, et al. Endoscopic management of biliary parasitic diseases. Dig Dis Sci. 2010 May;55(5):1472-8.

8. Borahma M, Afifi R, Benelbarhdadi I, Ajana FZ, Essamri W, Essaid A. Endoscopic retrograde cholangiopancreatography in ruptured liver hydatid cyst. Indian J Gastroenterol. 2015 Jul;34(4):330-4. 